\title{
Approximation of optical signals by the vortex eigenfunctions of the double finite Hankel transform
}

\author{
M.S. Kirilenko ${ }^{1,2}$

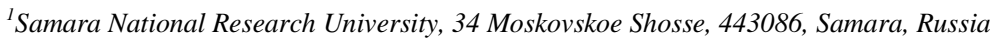 \\ ${ }^{2}$ Image Processing Systems Institute - Branch of the Federal Scientific Research Centre "Crystallography and Photonics" of Russian Academy of Sciences, 151 \\ Molodogvardeyskaya st., 443001, Samara, Russia
}

\begin{abstract}
This study considers a class of double finite Hankel transforms that describes the transmission of a vortex optical signal through a two-lens system with limited aperture radii in the object and spectral flats. The eigenfunctions of the given transform are calculated for different orders of vortex $\mathrm{m}$. The functions obtained are an orthonormal system of functions, which can help expand an unspecified limited optical allocation with high accuracy. Approximation of optical signals without radial symmetry is also performed in this paper.
\end{abstract}

Keywords: vortex optical beams; eigenfunctions; finite Hankel transform; approximation

\section{Introduction}

The current use rate of optical fiber in terms of time and frequency characteristics tends to the bandwidth limit [1]. However, the requirements to increasing the volume of global traffic are constantly growing. For the purpose of ensuring the correspondence of communication networks to ever-growing bandwidth requirements, additional approaches are considered for the multiplexing of optical fiber channels. One of such approaches is mode division multiplexing (MDM) [2,3]. Special advantage for increasing the bandwidth of the information channel is achieved with the help of optical beams with an orbital angular momentum and an infinite number of available quantum states [4]. The significant success of such method of channels multiplexing has already been demonstrated in optical fibers [5] and in free space [6,7]. For the purpose of forming and analysis of vortex beams, diffractive optical elements are used [8,9], and lens systems are used to place them into optical fiber [10, 11].

Transmission of a vortex laser beam of the $m^{\text {th }}$ order through the spherical lens can be described using the Hankel transform of the $m^{\text {th }}$ order. In real lens systems, there is a spatial limit, and finite (spatial-limited) transmission operators [12, 13] are used to describe the transmission of an optical signal. Due to the spatial limit both in the object and in the spectral region, it is impossible to obtain an ideal image in a two-lens system. In order to understand how the optical signal is distorted, it is necessary to expand them according to the eigenmodes of the lens system. As such, the concept of communication modes [14, 15] is widely used. Communication modes for square apertures and Fresnel transforms are prolated angulous spheroidal functions [16,17], which are widely examined and used in optics [18-21]. Communication modes for round apertures and finite Hankel transform are circular [22] and generalized [23] spheroidal functions.

The studies $[24,25]$ show the possibility of approximation of both one-dimensional and two-dimensional limited signals by spheroidal functions passing through the lens system without distortion.

For the purpose of superresolution development, the study [26] also examines spheroidal modes and generalized spheroidal functions, instead of which there were used the Zernicke polynomials in the calculation. For the record, the Zernike polynomials have a well-defined analytical form and they are often used in analysis problems of wave front and adaptive optics [27-29]. In contrast to the Zernike basis, spheroidal functions do not have an analytic representation and they are calculated as the eigenfunctions of an operator connected with a certain optical system. Expansion in the eigenfunctions of the system makes it possible to estimate the distortion of the transmitted signal in general, i.e. to assess the quality of information transmission by the system.

This study considers the transmission of optical signals through a two-lens imaging system based on a double finite Hankel transform of the $m^{\text {th }}$ order. We performed the calculation of an eigenfunctions set, which allows analyzing the distortion of an optical signal during transmission on the basis of approximation in functions of this set. Approximation of some signals with a good level of accuracy was performed.

\section{Brief theoretical information}

Let us consider the optical system in Figure 1. The optical beam passes through an aperture of radius $\mathrm{R}$ in the region $D_{1}$, , at the focus distance of which the lens is located. Then in the output focal plane of the lens (in the spectral plane $D_{2}$ ), it is located one more aperture of radius $P$. The output image of the beam is considered in the output focal plane of the second lens.

Let us consider vortex beams represented in the form:

$$
f(r, \varphi)=f(r) \exp (\operatorname{im} \varphi)
$$

ere $m$ is an integer number presenting the order of the optical vortex. 


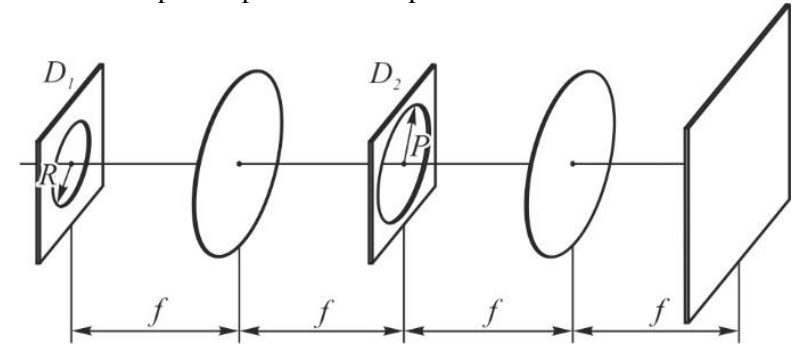

Fig. 1. Scheme of the optical system.

For vortex beams (1), the transmission of an optical signal through the two-lens system shown in Figure 1 can be written as follows:

$$
\begin{aligned}
& H_{R, P}[f(r) \exp (\operatorname{im} \varphi)]\left(r^{\prime}, \varphi^{\prime}\right)=\frac{k P}{2 \pi f} \int_{0}^{R} L\left(r, r^{\prime} ; m, P\right) f(r) r d r \cdot \exp \left(i m \varphi^{\prime}\right), \\
& L\left(r, r^{\prime} ; m, P\right)=\int_{0}^{2 \pi} \frac{J_{1}\left(\frac{k}{f} P \sqrt{r^{2}+r^{\prime 2}+2 r r^{\prime} \cos \varphi}\right)}{\sqrt{r^{2}+r^{\prime 2}+2 r r^{\prime} \cos \varphi}} \exp (\operatorname{im} \varphi) d \varphi,
\end{aligned}
$$

where $J_{l}$ - Bessel functions, $k$ - wavenumber, $f$-focus distance of both lens. Thus, if a vortex allocation is specified at the input of a given optical system, then there will be a vortex allocation at the output, as well, while the order of the vortex m does not change.

The eigenfunctions of the expansion operator $H_{R, P}$ represent an orthogonal system which it is used to expand optical signals that do not necessarily have a radial symmetry.

\section{Calculation of eigenfunctions and approximation}

The calculations will be performed with the following parameters: $k / 2 \pi f=1, R=1$ and $P=5$. Tables 1 and 2 show images of some functions and superpositions, respectively. The number $n$ corresponds to the number of the eigenfunction in the decreasing order of the moduli of eigenvalues.

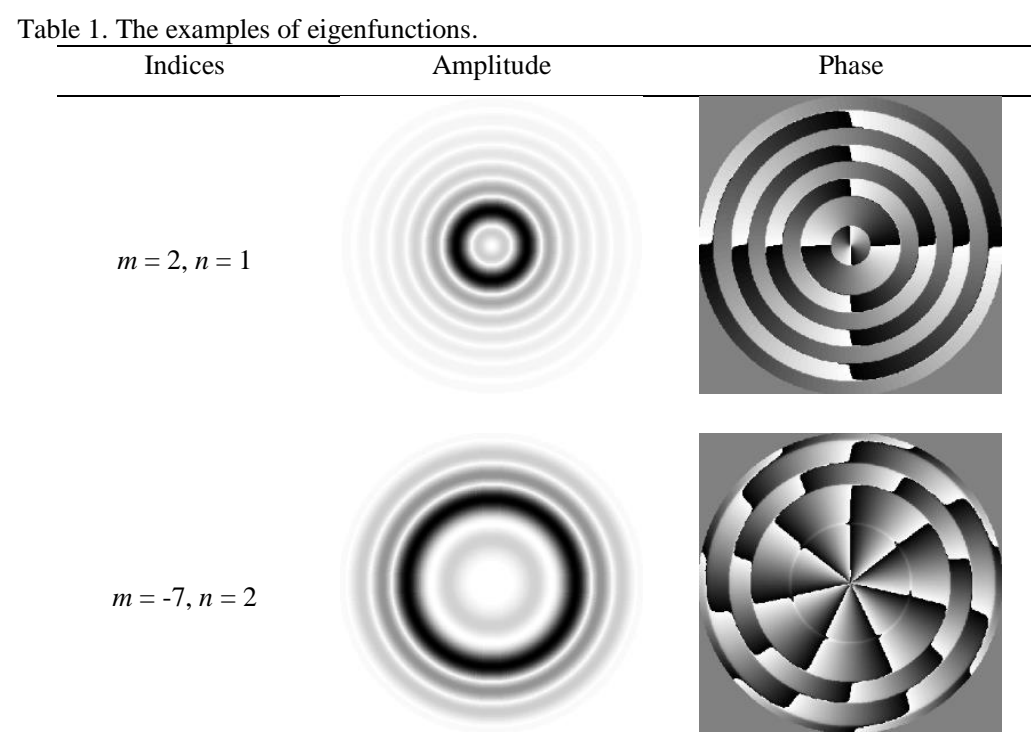

Table 2. The examples of superposition of eigenfunctions.

$\begin{array}{ccc}\text { Indices } & \text { Amplitude } \\ m_{l}=2, n_{l}=1 \\ + \\ m_{2}=-7, n_{2}=2\end{array}$




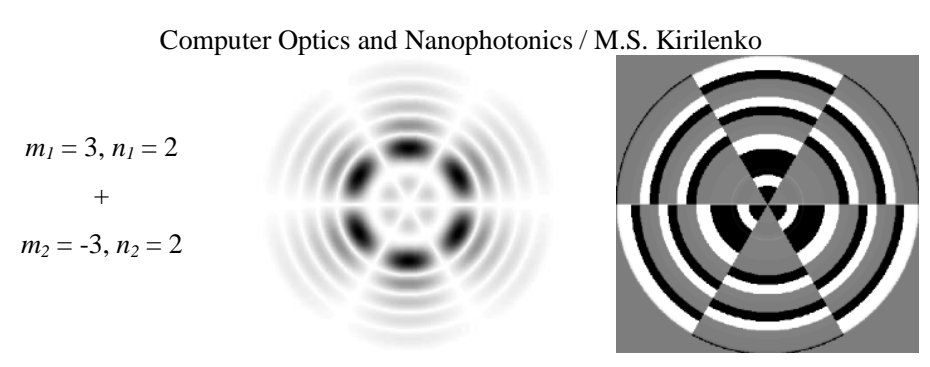

Table 3 shows which optical signals were approximated with the use of the calculated eigenfunctions. As such, there are approximation errors expressing the intensity standard deviation of an optical beam from its approximation by the eigenfunctions of the system. The expansion is performed only by those functions, which have the eigenvalue greater than 0.5 and in addition, $|m| \leq 8$.

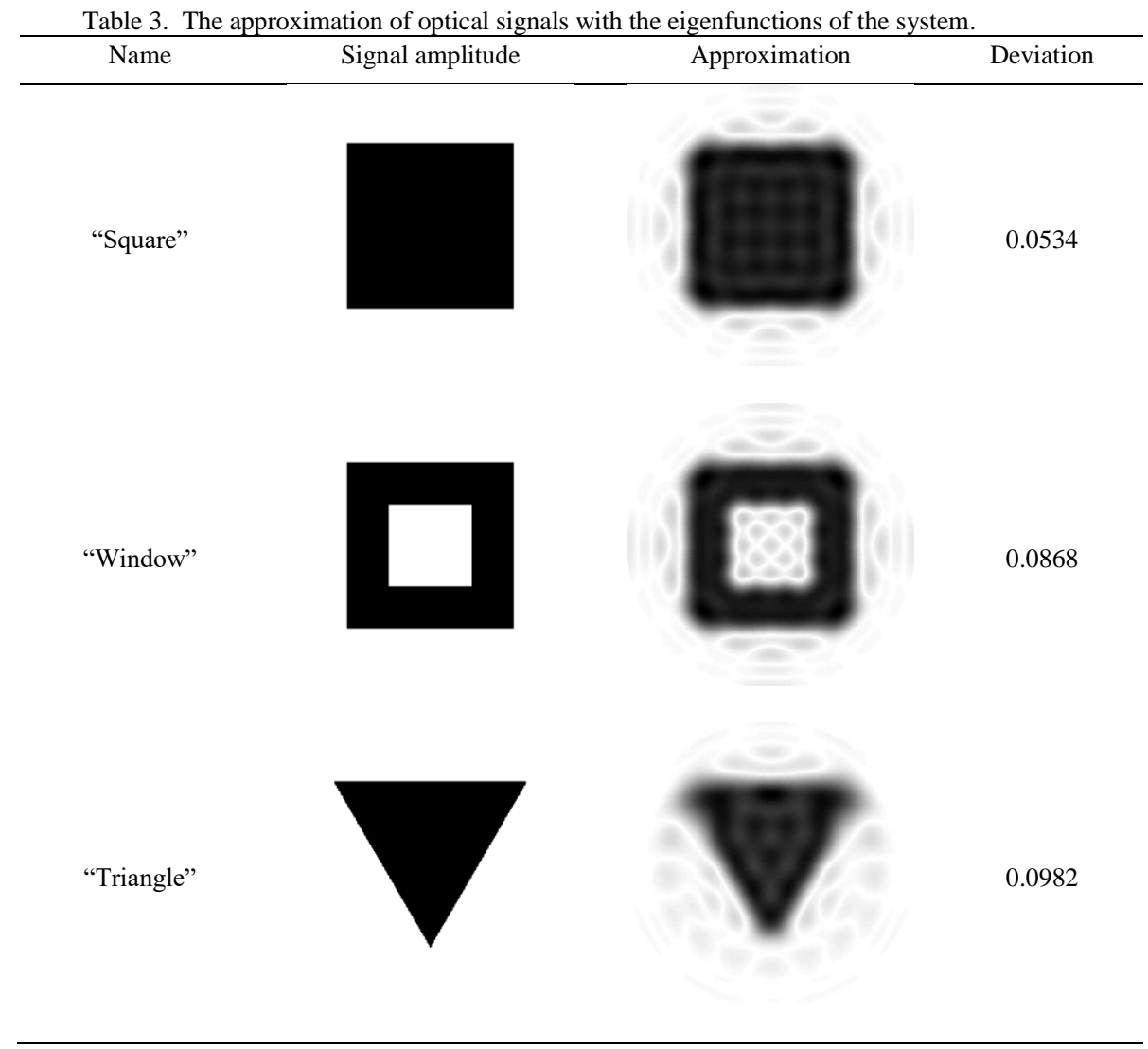

\section{Conclusion}

The calculation of the eigenfunctions of a given optical system is a complicated computational problem, especially at large values of the order of the vortices $m$. The operator $H_{R, P}$ written in the expressions (2) and (3) is self-adjoint, therefore its eigenfunctions must be real, and, consequently, their phase must be binary. However, the errors in the calculations introduce additional phase values.

Nevertheless, even in the presence of errors, the realization of the expansion of non-radial-symmetric signals along radialvortex eigenfunctions turned out to be possible, as it was demonstrated in this study. In this case, the deviations of the approximated functions from their originals were no more than $10 \%$.

It is worth noting that, for good approximation, the allocation of optical signals in the spatial region $D_{1}$ of the first aperture should not be larger than the dimensions of the aperture itself.

\section{Acknowledgement}

This study was conducted with partial financial support by the Russian Foundation for Basic Research (RFBR grants 16-47630546, 16-07-00825).

\section{References}

[1] Essiambre R, Kramer G, Winzer PJ, Foschini GJ, Goebel B. Capacity limits of optical fiber networks. J. Lightw. Technol. 2010; 28(4): 662-701. DOI: 10.1109/JLT.2009.2039464.

[2] Berdague S, Facq P. Mode division multiplexing in optical fibers. Appl. Optics 1982; 21: 1950-1955. DOI: 10.1364/AO.21.001950. 
[3] Khonina SN, Kazanskiy NL, Soifer VA. Optical Vortices in a Fiber: Mode Division Multiplexing and Multimode Self-Imaging. Recent Progress in Optical Fiber Research. Ed. by Dr. Moh. Yasin. Rijeka: InTech 2012; 327-352. DOI: 10.5772/2428.

[4] Soskin MS, Vasnetsov MV. Singular optics. Progress in Optics 2001; 4: 219-276.

[5] Bozinovic N, Yue Y, Ren Y, Tur M, Kristensen P, Huang H, Willner AE, Ramachandran S. Terabit-scale orbital angular momentum mode division multiplexing in fibers. Science 2013; 340(6140): 1545-1548. DOI: 10.1126/science.1237861.

[6] Yan Y, Xie G, Lavery MPJ, Huang H, Ahmed N, Bao C, Ren Y, Cao Y, Li L, Zhao Z, Molisch AF, Tur M, Padgett MJ, Willner AE. High-capacity millimetre-wave communications with orbital angular momentum multiplexing. Nature Communications 2014; 5: 4876. DOI: 10.1038/ncomms5876.

[7] Soifer VA, Korotkova O, Khonina SN, Shchepakina EA. Vortex beams in turbulent media: Review. Computer Optics 2016; 40(5): 605-624. DOI: 10.18287/2412-6179-2016-40-5-605-624.

[8] Khonina SN, Kotlyar VV, Soifer VA, Honkanen M, Lautanen J, Turunen J. Generation of rotating Gauss-Laguerre modes with binary-phase diffractive optics. Journal of Modern Optics 1999; 46(2): 227-238. DOI: 10.1080/09500349908231267.

[9] Khonina SN, Kotlyar VV, Soifer VA, Jefimovs K, Turunen J. Generation and selection of laser beams represented by a superposition of two angular harmonics. Journal of Modern Optics 2004; 51(5): 761-773. DOI: 10.1080/09500340408235551.

[10] Khonina SN, Karpeev SV. Excitation and detection of angular harmonics in an optical fiber using DOE. Computer Optics 2004; $26: 16-26$.

[11] Karpeev SV, Khonina SN. Experimental excitation and detection of angular harmonics in a step-index optical fiber. Optical Memory \& Neural Networks (Information Optics) 2007; 16(4): 295-300. DOI: 10.3103/S1060992X07040133.

[12] Sneddon IN. The Use of Integral Transforms. New York \& Boston: McGraw-Hill, 1972; 539 p.

[13] Debnath L, Bhatta D. Integral Transforms and their Applications, second ed. Boca Raton, FL: Goo Chapman and Hall/CRC Press, $2007 ; 778$ p.

[14] Miller AR. Communicating with waves between volumes: evaluating orthogonal spatial channels and limits on coupling strength. Applied Optics 2000; 39(11): 1681-1699. DOI: 10.1364/AO.39.001681.

[15] Martinsson P, Ma P, Burval A, Friberg AT. Communication modes in scalar diffraction. Optik 2008; 199(3): 103-111. DOI: 10.1016/j.ijleo.2006.07.009.

[16] Slepian D, Pollak HO. Prolate spheroidal wave functions, Fourier analysis and uncertainty - I. Bell System Technical Journal 1961; 40(1): 43-63. DOI: 10.1002/j.1538-7305.1961.tb03976.x.

[17] Landau HJ, Pollak HO. Prolate spheroidal wave functions, Fourier analysis and uncertainty - II. Bell System Technical Journal 1961; 40(1): 65-84. DOI: 10.1002/j.1538-7305.1961.tb03977.x.

[18] Khonina SN, Kotlyar VV. Effect of diffraction on images matched with prolate spheroidal wave functions. Pattern Recognition and Image Analysis 2001; 11(3): 521-528.

[19] Khonina SN, Volotovskii SG, Soifer VA. A method to compute eigenvalues of prolate spheroidal functions of zero order. Doklady Akademii Nauk 2001; 376(1): 30-32.

[20] Volotovskii SG, Kazanskii NL, Khonina SN. Analysis and development of the methods for calculating eigenvalues of prolate spheroidal functions of zero order. Pattern Recognition and Image Analysis 2001; 11(2): 473-475.

[21] Khonina SN. A finite series approximation of spheroidal wave functions. Computer Optics 1999; 19: 65-70.

[22] Karoui A, Moumni T. Spectral analysis of the finite Hankel transform and circular prolate spheroidal wave functions. Journal of Computational and Applied Mathematics 2009; 233: 315-333. DOI: 10.1016/j.cam.2009.07.037.

[23] Yoshinobu I. Evaluation of Aberrations Using the Generalized Prolate Spheroidal Wavefunctions. Journal of the Optical Society of America 1970; 60(1): 10-14. DOI: 10.1364/JOSA.60.000010.

[24] Kirilenko MS, Khonina SN. Coding of an optical signal by a superposition of spheroidal functions for undistorted transmission of information in the lens system. Proc. SPIE 2014; 9156: 91560J. DOI: 10.1117/12.2054214.

[25] Kirilenko MS, Khonina SN. Calculation of eigenfunctions for imaging two-lens system with axial symmetry. Computer Optics 2014; $38(3): 412-417$. DOI: 10.18287/0134-2452-2014-38-3-412-417.

[26] Pich'e K, Leach J, Johnson AS, Salvail JZ, Kolobov MI, Boyd RW. Experimental realization of optical eigenmode super-resolution. Optics Express 2012; 20(24): 26424-26433. DOI: 10.1364/OE.20.026424.

[27] Tyson RK. Principles of Adaptive Optics. Boca Raton, FL: CRC Press, Taylor and Francis Group, 2010; 314 p.

[28] Khonina SN, Kotlyar VV, Wang Ya. Diffractive optical element matched with Zernike basis. Pattern Recognition and Image Analysis 2001; 11(2): 442445.

[29] Porfirev AP, Khonina SN. Experimental investigation of multi-order diffractive optical elements matched with two types of Zernike functions. Proc. SPIE 2016; 9807: 98070E. DOI: 10.1117/12.2231378. 